\title{
An Investigation on the Concept of Triage in The Event of Disasters and Emergencies: Definition, Ethical Decision-Making
}

\author{
Hacer CANATAN ${ }^{1}$
}

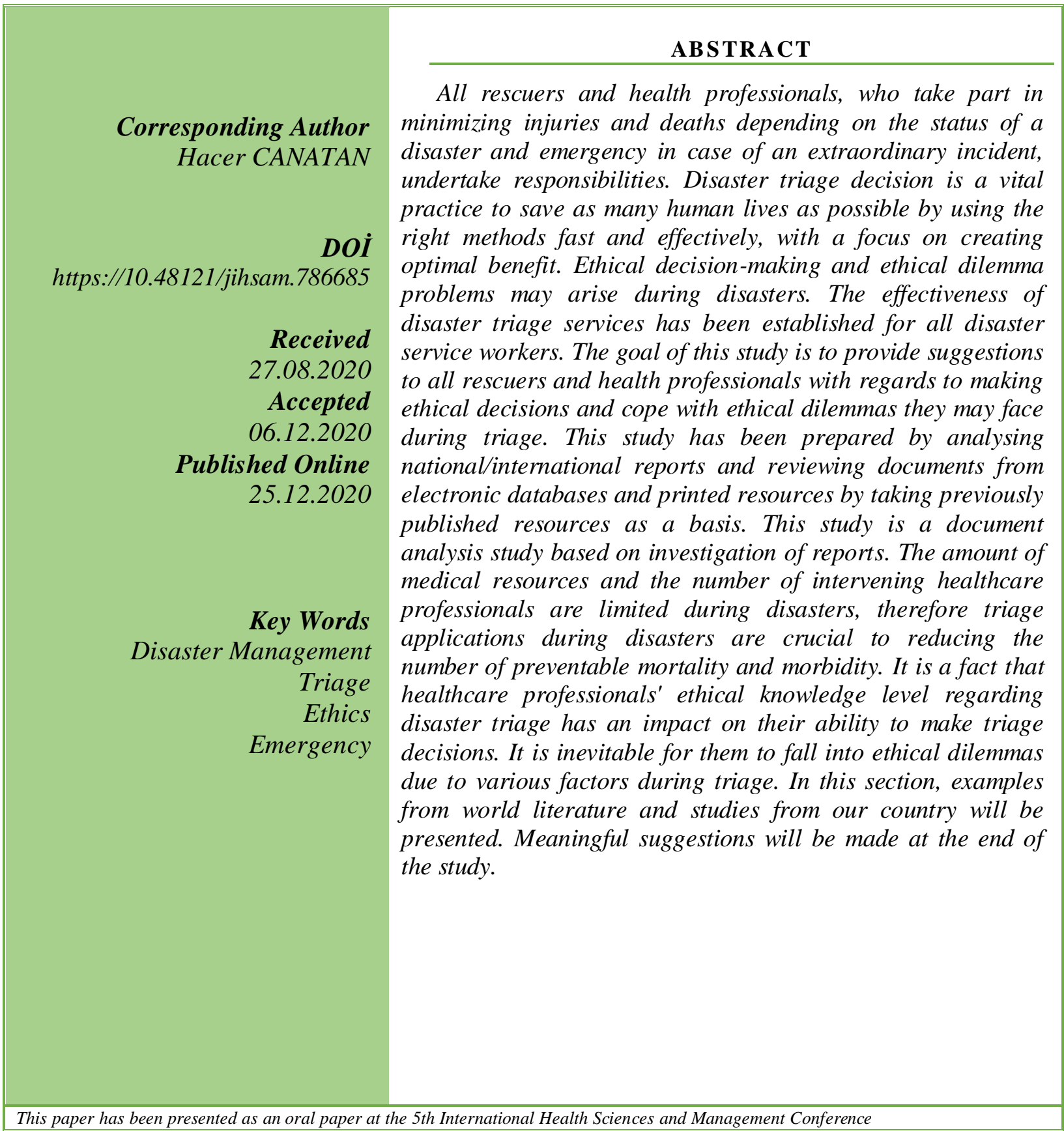

${ }^{1}$ Dr, Halic University / Faculty of Business Administration, Istanbul. hacercanatan58@ gmail.com. https://orcid.org/0000-0002-1406-3331 


\section{INTRODUCTION}

All rescuers and health professionals, who take part in minimizing injuries and deaths depending on the status of a disaster and emergency in case of an extraordinary incident, undertake great responsibilities. Disaster triage decision is a vital practice to save as many human lives as possible by using the right methods fast and effectively, with a focus on creating optimal benefit. The amount of basic medical resources and the number of intervening healthcare professionals are limited during disasters. Mortality and morbidity can be reduced by effective triage applications.

Ethical decision-making and ethical dilemma problems may arise during disasters. Theeffectiveness of disaster triage services has been established for all disaster service workers.

\section{MATERIALS AND METHODS}

The study is theoretical research based on literature review. This study has been prepared by analyzing national/international reports and national documents and reviewing documents from electronic databases and printed resources by taking previously published resources as a basis. This study is a document analysis study based on the investigation of reports.

\section{RESULTS}

It would be beneficial to define certain concepts used in the study. Definitions for the concepts of Emergency, Disaster, and Triage were taken from the Disaster and Emergency Management Presidency (AFAD,2014) Disaster Terms dictionary whereas the concept of Ethics has been defined based on Turkish Language Association (TDK, 2018)

Emergency: All situations and conditions that require urgency, usually at large scale but manageable with local resources. Law No. 5902 defines an emergency as an event that halts or interrupts normal life and activities for all or certain segments of society and requires immediate intervention as well as the state of crisis caused by such an event.

Disaster: Nature-, technology- or human-induced event that causes physical, economic, and social losses for all or certain segments of society, halts or interrupts normal life and human activities, and in which the capacity of the affected society to cope with is insufficient. A disaster is not the event itself but rather the consequence of it.

Triage: The process of quick selection and codification carried out at the scene or in healthcare facilities to which such individuals are admitted to identify individuals who require treatment priority or transfer in case of an event that causes multiple diseased or injured.

Ethics (ethique): As a noun; morals, the science of ethics, a set of behaviours that members of a profession must comply or abstain from. As an adjective; moral, related to morality.

\section{The Concept of Ethics and Ethical Dilemmas in Triage Decisions}

Ethics is the study of moral rules and judgments about what is right or wrong (Jenson, 1997:445). Ethics is the foundation of societies and cultures and constitutes the basis for political, social, and financial decisions. Ethics defines what is right or wrong, acceptable or unacceptable, tolerable, or not tolerable for an individual.

The study of ethics and the ethics theory is divided into two sections, namely metaethics and normative. (Fieser, 2009:1). In essence, metaethics questions the foundation, principles, and source of ethics and focuses on the meaning of terms related to ethics. It questions universal truths and locates the mind to judge it. Besides, normative ethics focuses on what moral standards and truths are to regulate behaviours.

Ethical dilemmas have sparked controversy throughout the years. According to Garrett Hardin (2001), "lifesaving ethics" is defined as difficulties faced in case of scarce resources. A boat with 50 people in it with enough capacity for 10 more people but with 100 people in water would be a good example. How to decide whom to save? Who should be allowed on board? Who will be left in the water? Certain decisions have to be made at that time. Another current example would be the ethical decision-making process during a pandemic in which medical resources are limited. Certain decisions are taken based on the principle of final justification. The decisions taken will be based on utilitarianism. It is not easy to evaluate an action based on its results Hardin (2001). We can never make a single decision; the truth is that an action will have multiple consequences while we are conducting our ethical analysis. When providing healthcare services; secrecy, confidentiality, and autonomy of those in need as well as the actions taken should be respected. There are multiple seriously ill and injured people and the situation requires enacting certain principles. Multiple individuals requiring care are present at the same time. The individuals to be treated first have to be determined. Limited available resources and the question of what the most appropriate use of them resulted in the emergence of the concept of triage. Triage, from the French word "trier", means ranking, eliminating, distinguishing, sorting (Streger, 1998:1). 
Triage was initially used to separate dying soldiers from those with lesser wounds on the battlefield.

Here, the decision-making mechanism was based on prioritizing the soldiers who were slightly wounded and therefore could heal quickly and return to the battlefield. In modern medicine, it means ranking patients based on their urgency (Williams, 1996:506). Ethical dilemmas may be experienced from time to time and a choice may have to be made among equally severe events and conflicting ethical rules (Jenson, 1997:8).

\section{History of Triage and Disaster Triage}

Before the concept of medical triage, triage was used as a tool to rate the quality of goods such as coffee beans and wool (Woolwich, 2000:476). The wars fought in history have laid the foundation for today's triage. The areas most needed in application fields of life-saving treatments according to patients' condition and the treatment they need such as accidents scenes before the hospitals, trauma centers of hospitals during disasters, emergence rooms, intensive care units, organ transplantations, and battlefields (Repine et al., 2005:505). Patients are initially received and assessed by emergency service healthcare personnel (such as nurses, paramedics, or emergency medical technicians). The staff in charge begins to life-saving procedures that prevent deterioration of the patient's condition while at the same time striving to lower mortality and morbidity in a multidisciplinary manner with doctor intervention and assessment.

Before the concept of medical triage, triage was used as a tool to rate the quality of goods such as coffee beans and wool (Woolwich, 2000:476).

The founder of the concept of medical triage was Baron Dominique Jean Larrey (1766-1842), Napoleon's surgeon (Howell, 1988:9). Triage means when and how to provide healthcare services at what level due to scarce resources in case of chaotic emergencies and disasters (Hogan and Burstein, 2007:7).

Soldiers condition would usually be ignored during the French Revolution as well as the war period of the 18th and 19th centuries. (Nestor, 2003:3). However, Baron Larrey, a dynamic and respected French military surgeon, revolutionized the treatment of soldiers on the battlefield in an era when surgeons were often perceived as butchers. With better care of the injured, Larrey was recognized as a good surgeon (Howell, 1988:9). Dominique Jean Larrey, the French war surgeon who was the first to apply the first triage practices, determined that a system for grouping soldiers injured in battle was necessary and started this procedure. He initiated basic medical care at the scene as soon as possible, thereby allowing them to recover quickly and survive. In 1846, John Wilson, a medical doctor in the English Navy, accepted the fact that better use of resources by way of treating those with most grievous wounds was necessary, which was the next major development in triage systems (Hogan and Lairet, 2007:17).

During the American Civil War, Jonathan Letterman, medical director of the Potomac Army, initiated triage and frontline medical care which resulted in a significant reduction in death rates of privates in his unit (Christian et al., 2002:5). Horse carts called flying ambulances or "Les ambulances valantes" (Figure 1) would be used to evacuate injured soldiers who are likely to survive from the battlefield and bring them to treatment areas (Mitchell, 2008:5).

\section{The ambulance corps of Baron Dominique-Jean Larrey, circa 1809.}

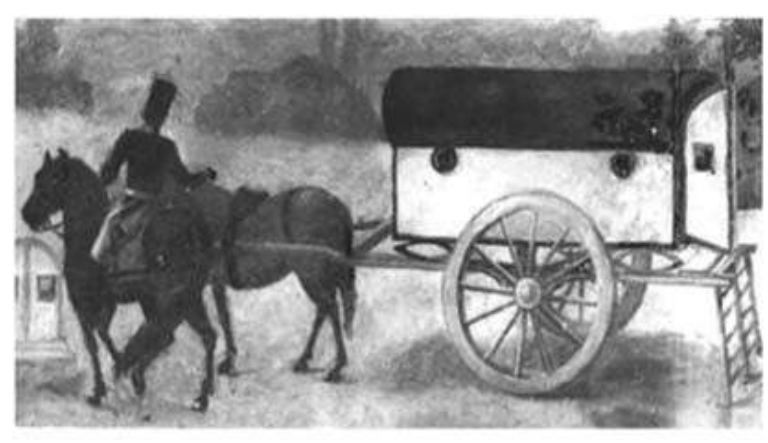

Figure 1

During World War One, the wounded would be transported to a location that was turned into a treatment center and then directed to appropriate centers for advanced care.

During World War One, ambulances comprised of civilian volunteers were at the forefront; they would stand right behind the frontlines and many wounded soldiers were evacuated by ambulance vehicles while the fighting continued, as shown in Figure 2 (Mitchell, 2008: 5).

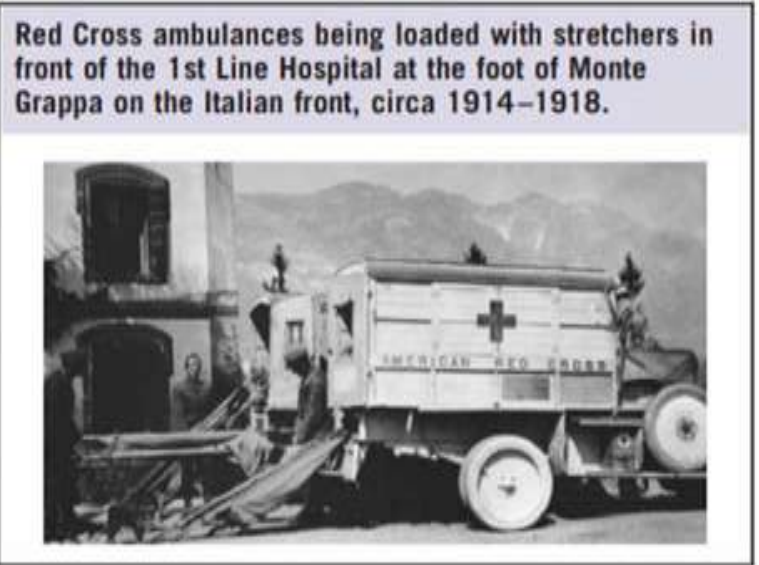

Figure 2 
The use of chemicals and machine guns in combat resulted in a large number of injuries, forcing paramedics to use triage techniques. In order to optimize overall patient outcomes in a catastrophic situation, there is a shift from doing what is best for the individual patient to doing the greatest good for the largest number of people The war now involved civilian population centers; triage spread throughout the medical community and medical staff had to prioritize treatment of civilian casualties (Lee,2010:466)

Significant advancements were made in the field of medicine during World War Two; the concept of "Buddy Aid" emerged and each soldier was provided a package of medical items and a tourniquet for first aid on himself and his teammates. The doctor was an integral part of each combat unit, and they would aid the injured who shouted "Medic!" Contents of a small medical aid bag allowed initial treatment which enabled many soldiers, who would otherwise die of blood loss or chest wounds, to survive. Today, the stepped treatment has been developed to treat injuries. Treatment is initiated by healthcare professionals in the field; afterward the individual transitions into necessary advanced treatment. Badly injured soldiers were brought into nearby aid stations (Mitchell, 2008:4). The surgical hospitals allowed treatment of many surgical wounds which would be fatal (Dolev, 1996:785). Later, surgeons were moved away from the conflict zones to operate in a safe environment. The emerging aviation technology would be used for quick evacuation of injured soldiers; a helicopter was launched as shown in Figure 3. (Mitchell, 2008:5). This allowed lowering mortality rates during wartime to below $30 \%$.

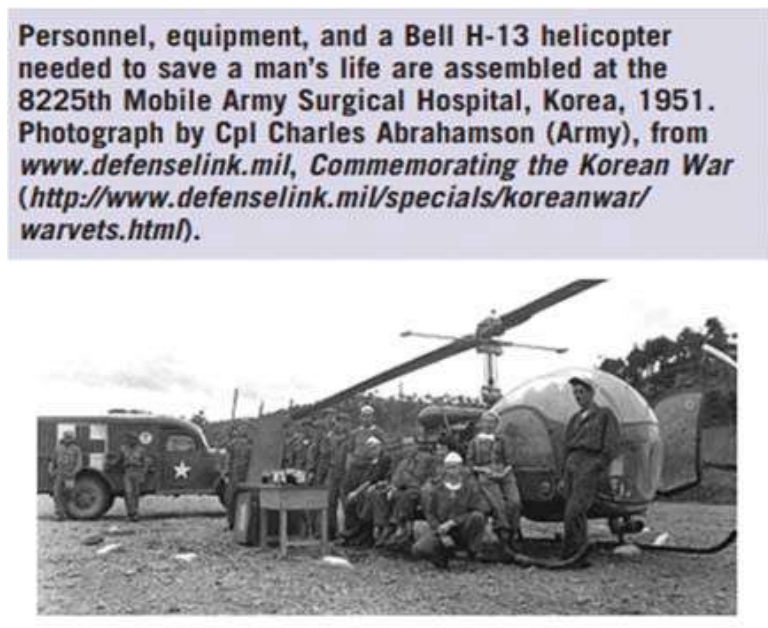

Figure 3

Advancements in helicopter evacuation have revolutionized wartime medical care. This allowed providing quick medical care to soldiers who would otherwise die in conflicts (Eisman, 1967, 153).

Mortality due to injuries in the battlefield reduced to $24 \%$ in Vietnam and $10 \%$ in Iraq and Afghanistan. Triage became what it is today after the Vietnam War
(Mitchell, 2008:6).

This change in approach to triage was one of the factors that influenced instant life save during the Korean War and the Vietnam War, during which fast triage and evacuation system was further improved. The goal of military triage was to treat the injured at higher rates, whereas civilian triage was focused on keeping as many people alive as possible (Nocera and Garner, 1999:603). The disaster medicine was developed as a method of coping with a high number of injured people. Many scientists have adopted advanced triage plans; however, few of these could be used in case of a disaster. Triage was developing in the scientific sense but the US government has focused on the development of advancement of designed triage systems after the 9-11 attacks Triage has become an integral part of various medical fields; even approaches towards the quick treatment of slightly wounded individuals, such as firemen and policemen, to have them return to duty have been developed. A coordinated and multidisciplinary triage approach is suggested for effective triage functions in case of mass injuries (Mitchell, 2008:5).

Triage in case of mass accidents or incidents such as derailed trains is different from triage used in case of extraordinary events. This table is part of a healthcare facility's daily work system. It requires Incident Command Management and the process can be managed with good planning. Triage used in emergency services or healthcare facilities daily is focused on treating individuals with the most severe illness or those requiring urgent care. This group of people has to be prioritized for life-saving treatments. Advanced techniques are applied to increase patients' probability of survival.

Disaster triage differs significantly from triage used in the emergency rooms daily. Patients who apply to the emergency department are assorted based on urgency and are treated. Every patient who presented to emergency room can receive medical care and be treated.

In case of mass casualties such as disasters, the goal of triage is to quickly classify individuals to save as many lives as possible and this is where we come across the concept of utilitarian ethics The system ensures that scarce resources go to those who will benefit the most from the most appropriate care and rapid surgical intervention. Ethical difficulties might occur during disasters due to insufficient or disorganized resources, limited time, a high number of injuries, or a chaotic environment. Nevertheless, the individual providing healthcare services combines the basic ethics of respect for autonomy and fairness with his own ethical rules and his behavior takes shape accordingly (Bosticks, 2008:35).

Disasters create a large portion of aid efforts; the management requires an institutional approach (Cariappa, Khanduri, 2003:286). Many people try to work in an alien environment and at a speed with 
which they would not be able to keep up under normal conditions (Auf Der Heide, 1984: 4). Because disasters cause never before seen, extraordinary problems and people try to solve these problems. Disasters are events that occur suddenly and require timely intervention to mitigate the damage caused by unfolding events.

There are many disaster triage systems around the world, each of them aims to do the best for as many injured people as possible (Brosnan et al., 2010). Triage systems developed for use in non- military systems are divided into two groups, namely primary and secondary triage. In primary triage systems, injured people are prioritized for evacuation, they are transferred to medical centers for medical care (Brosnan et al.,2010:178).
In secondary triage systems, the injured people are triaged after arriving at the scene; on-scene treatment is provided if evacuation is going to delay. (Jenkins et al., 2008:138).

\section{Triage classification is based on the condition} of injuries.

START triage system: This system is the most commonly used triage system in the United States. In this system, all injured adults older than 8 years are evaluated, based on the algorithm of the system in 60 seconds or less (preferably 30 seconds). In this system, the criteria including the ability to walk, respiratory rate, capillary filling, radial pulse and obeying the commands are used. By examining each criterion, the patient will be marked by one of the red, yellow, green and black tags (Figure 4).

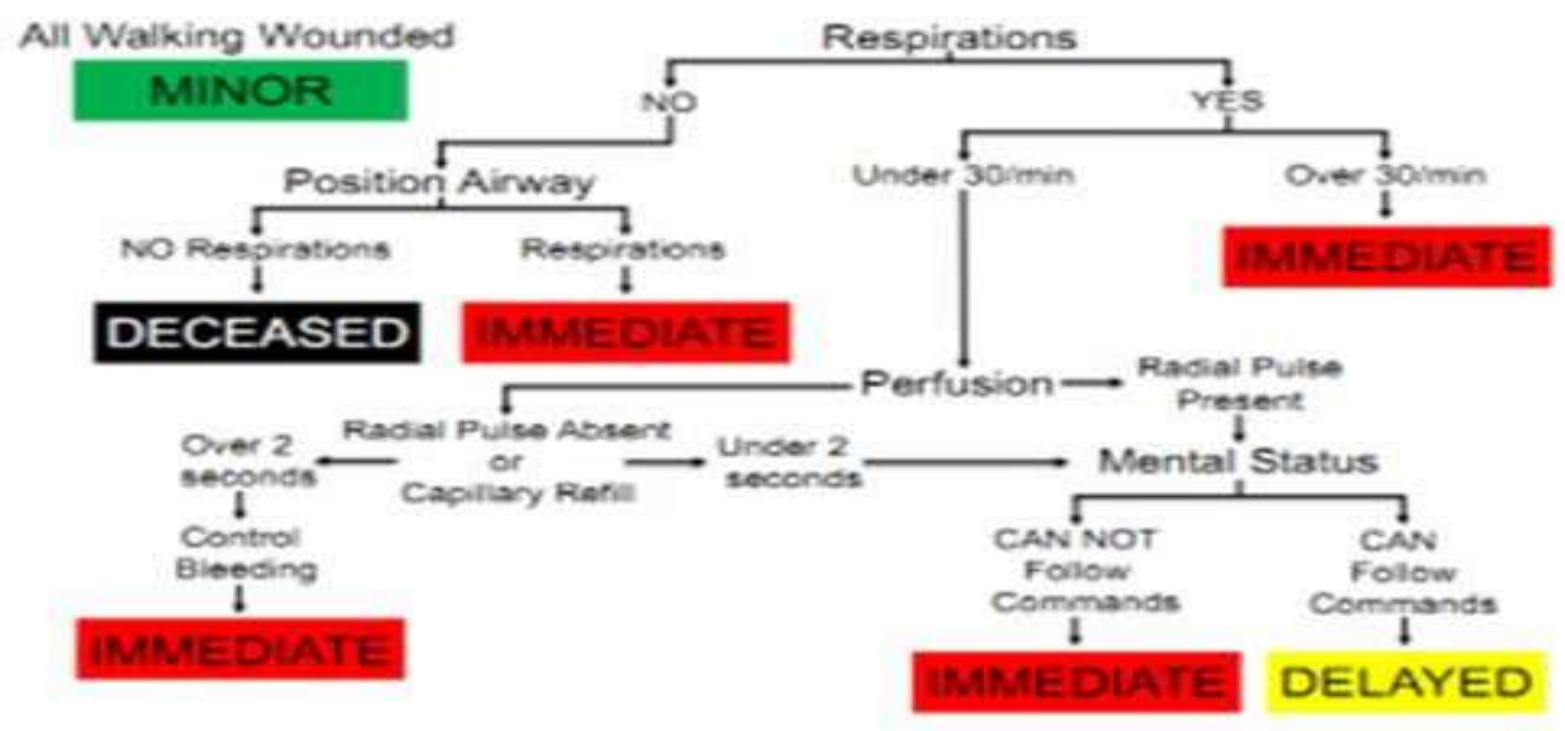

Figure 4. START Triage Algorithm (Bhalla et al.,2015:7)

Reverse Triage: Reverse triage is a method that is commonly used during emergencies and disasters. In reverse triage, injured people with fewer damages and minor injuries are at the priority of receiving services. This is also used in cases, where the treatment team or soldiers, during the war, are injured. Moreover, this kind of triage system is used in the disaster and emergencies, where medical resources are limited, with the aim of returning people as quickly as possible and helping other people (Jenkins at al.,2008:5).

Military Triage: The main goal of the military triage is to treat and return more injured soldiers to the battlefield. In this method, immediate and rapid classification of the injured people is based on the type and severity of the injury, the probability of survival, as well as the priority of treatment in order to provide the best health care services for the largest number of people (Adams,2008:216).

Sieve Triage: Similar to the START method, this method, which is used in parts of Europe, Australia, and the United Kingdom, first uses the walking filter to examine the injured individual, and uses four tags encompassing red, yellow, green and black tags to classify the injured patients (Figure 5). 


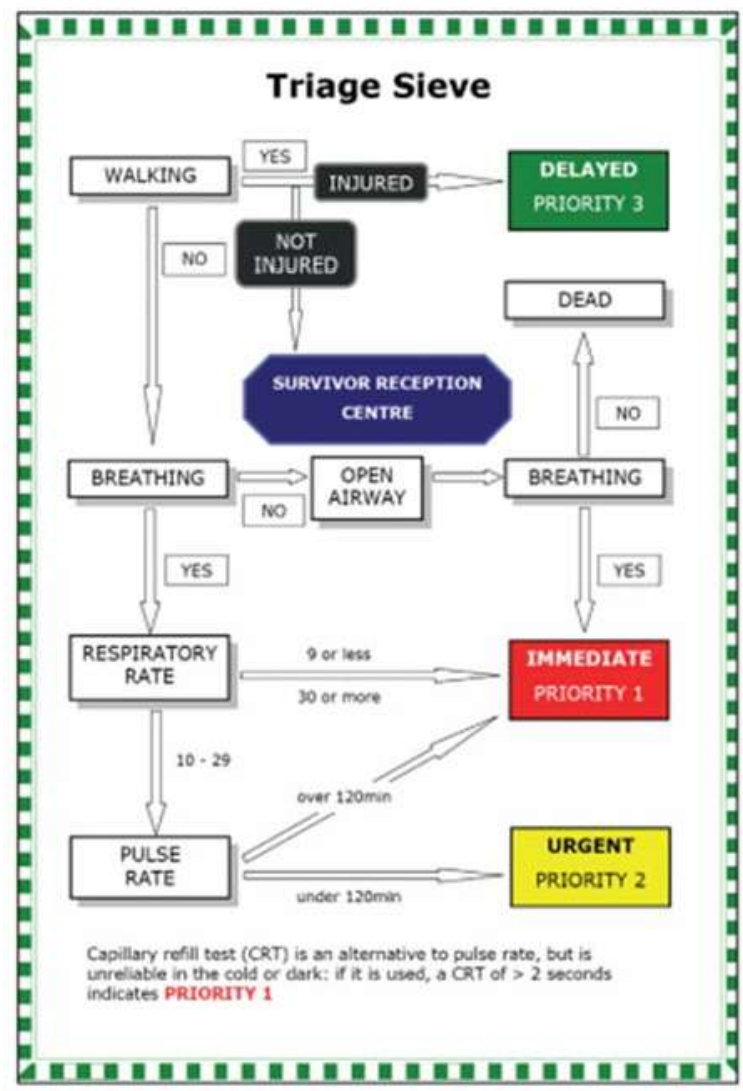

MASS triage (Move, Assess, Sort, Send): This system is a disaster triage system used in the United States. Although this system is based on the START triage system, it does classify the injured people before individual examination. This includes four stages of moving, evaluating, classifying and transferring. This system, whose algorithm is very and green classes. The red class includes people, who are unconscious and in shock, have bleeding, and ineffective breathing. The yellow class involves patients with fractures of the bones and other injuries, and the green class includes injured people, who can walk (Figure 6).

Figure 5. START Triage Algorithm (Smith, 2012:414)

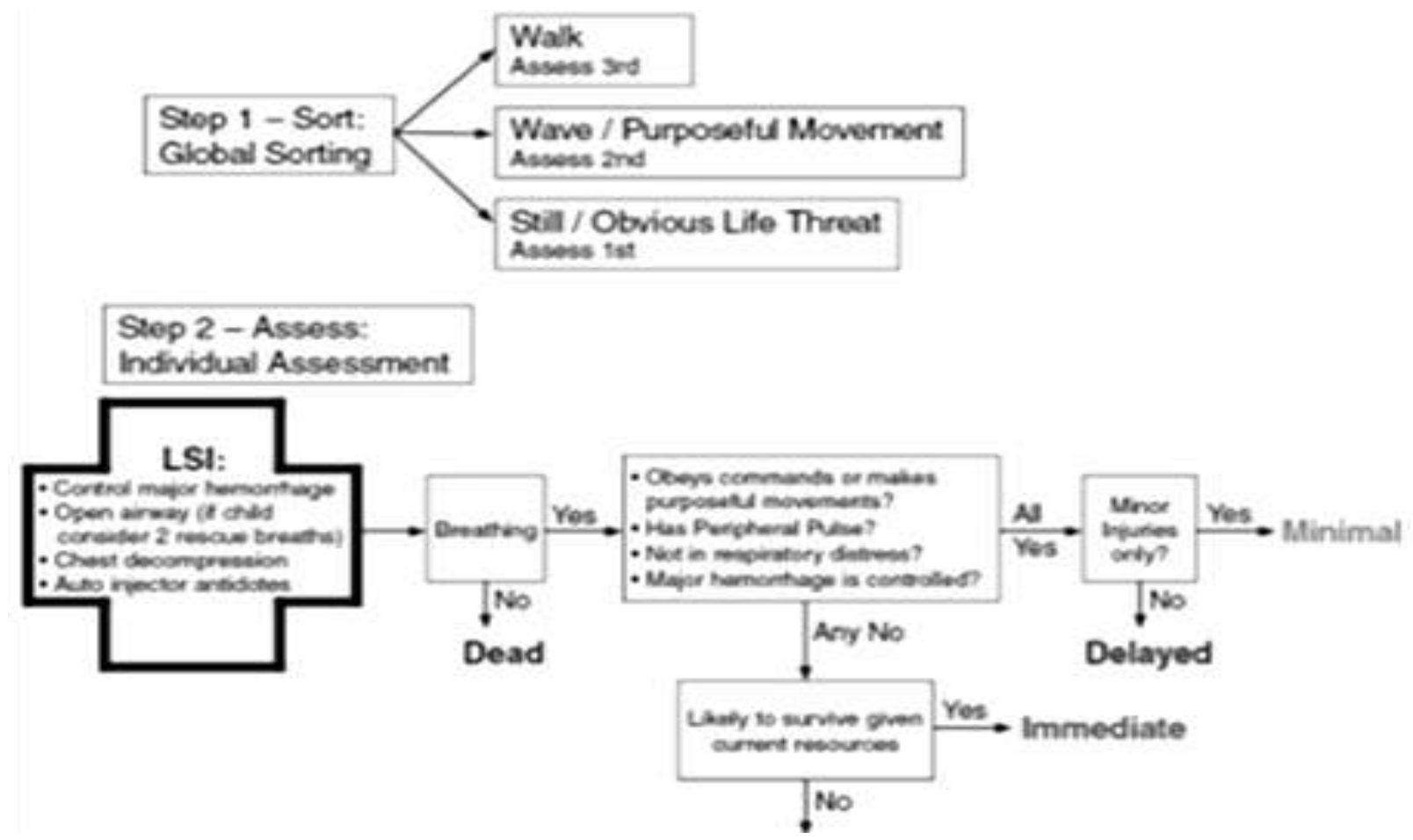

Figure 6. Proposed national triage guideline: SALT mass casualty triage (Lerner,2008:35)

Burn Triage: In this method, which is used to prioritise injured persons in burn events, the classification of the injured people is based on the severity and level of the burn (Figure 7). 


\begin{tabular}{ll}
\hline Category & Profile \\
\hline Green group & First- degree and superficial burns \\
Yelow group & Burns above $30 \%$ in people over 5 and \\
under 60 years old & Second. degree burns in head and neck, \\
Red group & genital area and joints \\
& Third-degree burns in an anatomical region \\
& of the body \\
Burn in people under 5 years of age and \\
over 60 years of age \\
Burn in pregnant wornen, people with \\
undertying oonditions with socond. degree \\
burns more than $10 \%$, people with second- \\
degree burns above $30 \% 6$
\end{tabular}

Figure 7. Classification of the injured people in the Burn triage (Brandt et al., 2000: 26).

Medical Triage Protocol: In this protocol, the walking ability criterion is initially controlled, and those who can walk are classified in the green group. Then, other criteria such as the level of consciousness, arterial bleeding, shock, breathlessness, fractures and injuries of the head and spine, and ultimately pathologies such as myocardial infarction, poisoning, burns, hypothermia, and chest pain are checked and the patient is tagged as red or yellow according to the following algorithm (Figure 8).

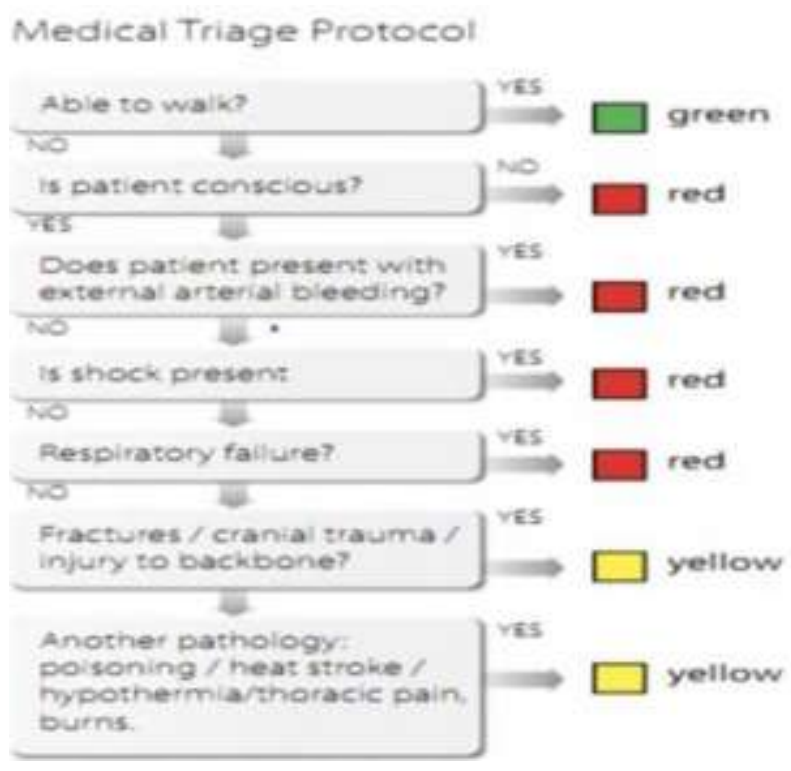

Figure 8. Medical Triage Algorithm (Alexander, 2013:27)

Hospital Triage: The aim of the hospital triage in the emergency department is to place patients in a suitable clinical setting at the right time to receive the appropriate level of health care. There are two, three, four, and five level systems for hospital triage proposed in the world, among which five-level systems including Manchester Triage System (MTS), Canadian Triage and Acuity Scale (CTAS), Australia Triage System (ATS). All hospitals should design and develop a program for hospital triage in disaster situations and mass casualty incidents as part of the hospital emergency plan (Powers, 2010:10) Regardless of the system used, triage requires ethical decision-making. Disaster triage is utilitarian by nature (Hogan and Burstein, 2007:8). In cases of disasters, triage is an ongoing process. It starts at the scene and continues when you arrive at a healthcare facility. Each process within the care system of a healthcare facility such as surgery, intensive care admission, or discharging patients requires medical teams to make ethical decisions. Healthcare professionals' failure to treat injuries based on the decision taken due to low resources or prioritization is not considered as a failure of duty. The goal here is to provide as much benefit as possible based on the approach of providing a benefit by using limited resources. Mill used this as the functional basis of ethics (Mill, 1867:8).

Healthcare professionals may act slowly, be late for saving lives or breach the principle of not harm by disregarding the fact that time is of vital importance (Demirhan,2009:28). Over the years, sets of ethical rules have been developed to guide medical personnel in their work. Ongoing debates over triage decisions based on age, gender, social status, ethnicity, or profession (e.g. healthcare workers) of victims generally conflict with the basic right of survival at the individual level as well as the principle of fairness. In their study, Halpern and Larkin concluded that "ideological issues should not overshadow human priorities embodied by ethical rules" They regard the patient's health as being the first priority for the physician, who is mandated to use the power of medicine forthe general good and maintain good relationships with his or her colleagues, while forbidding any kind of discrimination among patients (Halpern et al, 2006:63).

Another important issue is the fact that disaster victims may reject treatment while healthcare professionals working in the disaster areas are trying to initiate and continue treatment according to triage rules. In such cases, treatment should continue to avoid medical or legal problems if the victim's ability to make decisions is doubtful while assessing his/her 
mental capacity. Victims who have been grievously wounded during a disaster may demand euthanasia. Euthanasia is prohibited under international public law and most medical ethics around the world (Massue JP,2000:462). This prohibition was declared by the WMA in the Declaration of Euthanasia (1987) stating euthanasia is not ethical (WMA, 1987). Health workers working in disasters should particularly pay attention to vulnerable groups; such groups including children, women, the elderly, the disabled, refugees, and other minority groups, all of which are defenseless and therefore affected more severely by disasters. (UNDP, 1997). Besides, any activity that may result in stigmatization or discrimination against vulnerable groups should be avoided based on the principle of ethical fairness (Karadağ et al, 2012: 609).

\section{CONCLUSIONS}

The results obtained from literature research and review show that disaster triages cause ethical dilemmas due to crumbled weak structures in disaster areas and a high amount of victims who were buried in the wreckage as well as lack of current resources and personnel trained to use such resources. Disasters will continue to occur and certain ethical issues and dilemmas will accompany them.
Disasters will occur in different shapes based on time, location, the affected area, and the number of affected people, therefore the ethical issues that arise will not be uniform.

Establishing ethical values and principles in each area where healthcare services are provided in case of a disaster is crucial.

\section{RECOMMENDATIONS}

The ultimate goal of triage is the preservation of lives. A group of healthcare professionals, who have the skills and dynamic structure to create the most benefit with limited resources in case of a disaster and the midst of a chaotic environment, should be trained. The presence of a group with disaster medicine and war surgery skills is important. It is suggested that the formation of professional medical teams similar to those previously present in the military healthcare system would be beneficial.

Having experienced experts provide training, conducting drills, and enacting scenarios are suggested to develop healthcare professionals' ethical decision-making and ethics-based triage application skills during triage training. Also, it is suggested that having the trained teams engage in missions for actual exercises in the event of a disaster would contribute to the teams' training and development.

Establishing a legal definition for the ethical disaster triage practices deemed fit for use in case of a disaster that occurs in our country as well as detailing it, is suggested as it would enable us to react to various problems that may occur following a disaster.

It is suggested that inefficient practices during any disaster should be criticized and recorded, as it would be beneficial in initiating corrective/preventive actions in later stages.

It is suggested that developing guides, protocols, and emergency/disaster relief plans by reviewing legal and institutional regulations and taking possible ethical dilemmas into considerations, creating on-call ethical committees and giving sufficient in-service ethical competence training to healthcare professionals would be beneficial as steps to be taken before any disaster.

It is suggested that discussing reformatory efforts at the national and international level would provide a holistic benefit.

\section{REFERENCES}

Adams MP. Triage priorities and military physicians. In Physicians at War. Springer, Dordrecht, 2008. https://doi.org/10.1007/978-1-4020-6912-3_13 PMid:18421142 PMCid: PMC2394804.

AFAD. (2014). Açıklamalı Afet Yönetimi Terimleri Sözlüğü. Ankara: T.C. Başbakanlık Afet ve Acil Durum Yönetimi Başkanlı̆̆ı.

Alexander D, Masini E, Mugnai L. Integrated Emergency Management for Mass Casualty Emergencies, 2013.

Auf Der Heide, E. (1989), "The apathy factor", Disaster Response: Principles of Preparation and Coordination, St. Louis Mosby, pp. 1-303, available at: http://orgmail2.coedmha.org/dr/flash.htm.

Bhalla MC, Frey J, Rider C, Nord M, Hegerhorst M. Simple Triage Algorithm and Rapid Treatment and Sort, Assess, Lifesaving, Interventions, Treatment, and Transportation mass casualty triage methods for sensitivity, specificity, and predictive values. Am J Emerg Med. 2015; 33(11):1687-91. https://doi.org/10.1016/j.ajem.2015.08.021 PMid:26349777.
Bostick, N. A., Subbarao, I., Burkle, F. M., Hsu, E. B., Armstrong, J. H., \& James, J. J. (2008). Disaster triage systems for large-scale catastrophic events. Disaster medicine and public health preparedness.

Brandt C, Coffee T, Yurko L, Yowler C, Fratianne R. Triage of minor burn wounds: avoiding the emergency department. The Journal of burn care \& rehabilitation. 2000. https://doi.org/10.1097/00004630-200021010-00006.

Brosnan, D.P., Kahn, C.A., Brooke, E.L. and Cone, D.C. (2010), "Triage", Koening and Schultz's Disaster Medicine Comprehensive Principles and Practices, Cambridge University Press,NY.

Christian, M.D., Farmer, J.C. and Young, B.P. (2002), "Disaster triage and allocation of scarce resources", accessed at: www.sccm.org.

Dawkins, F.W. (2006), "Good samaritans or mercy killers: and never the twain shall meet", available at: www.law.uh.edu. 
Demirhan EA. The ethical and deontological problems in emergency treatment and care in Turkey. The Turkish Annual of the Studies on Medical Ethics and Law 2009-2010.

Dolev E. Ethical issues in military medicine. Israel J Med Sci. 1996. Eiseman B. Combat casualty management in Vietnam. J Trauma. 1967.

Fieser, J. (2009). Ethics. In Internet Encyclopedia of Philosophy. Retrieved from http://www.iep.utm.edu/ethics/

Halpern P, Larkin GL. Ethical Issues in the Provision of Emergency Medical Care in Multiple Casualty Incidents and Disasters. In: Ciottone GR, editor. Disaster Medicine. 3rd ed. Philedelphia: Elsevier Mosby; 2006.

Hogan, D.E. and Burstein, J.L. (2007), "Basic perspectives on disaster", in Hogan, D.E. and Burstein, J.L. (Eds), Disaster Medicine, Lippincott Williams \& Wilkins, Philadelphia, PA.

Hogan, D.E. and Lairet, J.R. (2007), "Triage”, in Hogan, D.E. and Burstein J.L. (Eds), Disaster Medicine, Wolters Kluwer/Lippincott Williams \& Wilkins, Philadelphia, PA.

Howell, E. 1988. Foundation for trauma nursing, in Comprehensive trauma nursing: theory and practice, edited by $\mathrm{E}$ Howell. L Wildra \& M Gail Hill. Glenview, III.: Scott \& Foresman.

http://www.garretthardinsociety.org/gh/gh

straub_interview.html. Living in a World of Limits - An interview with noted biologist Garrett Hardin by Craig Straub, The Social Contract, Fall, 1997.

Jenkins JL, McCarthy ML, Sauer LM, Green GB, Stuart S, Thomas TL, et al. Mass-casualty triage: time for an evidencebased approach. Prehospital and disaster medicine. 2008. https://doi.org/10.1017/S1049023X00005471 PMid:18491654

Jenson, E. (1997), "Disaster management ethics (training program)", available at:www.humanitarianinfo.org.

Lee $\mathrm{CH}$. Disaster and mass casualty triage. The Virtual Mentor :VM. 2010 ;12(6):466-470. DOI: 10.1001/virtualmentor.2010.

Lerner EB, Schwartz RB, Coule PL, Weinstein ES, Cone DC, Hunt RC, et al. Mass casualty triage: an evaluation of the data and development of a proposed national guideline. Disaster medicine and public health preparedness. 2008. https://doi.org/10.1097/DMP.0b013e318182194e PMid:18769263
Massue JP. Ethics and Disaster Medicine: Fundamental Concepts. In: de Boer J, Dubouloz M, editors. Handbook of Disaster Medicine. Amsterdam: International Society of Disaster Medicine; 2000

Mitchell, G. W. (2008). A brief history of triage. Disaster Medicine and Public Health Preparedness, 2, pp.4-7. doi:http://dx.doi.org/10.1097/DMP.0b013e3181844d43

Mill, J.S. (1867), Utilitarianism, 3rd ed., Longmans, Green, Reader and Dyer, London.

Nestor, P. 2003. Triage history. [Online]. Available: journal of Emergency Primary Health Care,

1 (3-4): (Article Number: 990004)

Nocera, A. and Garner, A. (1999), "An Australian mass casualty incident triage system for the future based upon triage mistakes of the past: the homebush triage standard", Aust. N. Z. J. Surg., Vol. 69

Powers R, Daily E. Disaster triage. International disaster nursing New York: Cambridge Universiry Press, 2010 https://doi.org/10.1017/CBO9780511841415

Repine, T.B., Lisagor, P. and Cohen, D.J. (2005), "The dynamics and ethics of triage: rationing care in hard times", Military Medicine, Vol. 170 No. 6, pp. 505-9. DOI: $10.7205 /$ milmed.170.6.505

Smith W. Triage in mass casualty situations. Continuing Medical Education. 2012. Streger, M. R. (1998). Prehospital Triage. Emerg Med Serv.

TDK. (1988). Türkçe Sözlük. Ankara: Türk Tarih Kurumu Basımevi

United Nations Development Programme, Disaster Management Training Programme. In: Jenson E, editor. Disaster Management Ethics. 1st ed. UNDP; 1997.

Williams, R. M. (1996). Triage and Emergency Department Services. Annals of Emergency Medicine, 27(4), 506-508.

World Medical Association. WMA Declaration on Euthanasia. 39th World Medical Assembly; 1987 Oct; Madrid, Spain. (reaffirmed in 2005). [cited 2020 July 27]. Available from: http:// www.wma.net/en/30publications/10policies/e13/

Woolwich, C. 2000. Accident and emergency: theory into practice. London: Tindall. 